\title{
Effect of ultrasound exposure on the Triticum aestivum root elongation test
}

\author{
IULIA MARINA POTOLEA ${ }^{1}$, OANA MARINA PIRVU ${ }^{1}$, CRISTIANA ELENA SPINU ${ }^{1}$, \\ DRAGOŞ PAUL MIHAI ${ }^{1}$, OCTAVIAN TUDOREL OLARU ${ }^{1}$, GEORGE MIHAI NIŢULESCU ${ }^{1}$, \\ ARISTIDIS TSATSAKIS ${ }^{2}$, DEMETRIOS A. SPANDIDOS ${ }^{3}$ and GEORGIANA NIŢULESCU ${ }^{1}$ \\ ${ }^{1}$ Faculty of Pharmacy, 'Carol Davila' University of Medicine and Pharmacy, 020956 Bucharest, Romania; \\ ${ }^{2}$ Department of Forensic Sciences and Toxicology; ${ }^{3}$ Laboratory of Clinical Virology, \\ School of Medicine, University of Crete, 71003 Heraklion, Greece
}

Received October 2, 2019; Accepted November 13, 2019

DOI: $10.3892 /$ wasj.2019.27

\begin{abstract}
The Triticum aestivum root elongation test can be used as a simple and effective method for the identification of novel potential anti-proliferative agents. The employment of this method is based on the fact that a significant number of oncogenic signal transduction pathways are highly conserved in living organisms. Moreover, the use of phytobiological assays has several advantages, such as rapid outcomes, simple procedure and reduced costs. Previous studies have demonstrated that ultrasound exposure can have lethal effects on small organisms, medium and high frequency exposure, leading to cell destruction. In the present study, the effects of ultrasound exposure on the radicular length of Triticum caryopses were analyzed as a potential tool for further experimentation using ultrasounds as a stress model in phytobiological tests. The influence of the exposure time was determined at different intervals and was proven to exert significant effects. Short intervals of $<5$ min produced no statistically significant effects on root elongation, and the maximum of inhibition was achieved following $30 \mathrm{~min}$ of exposure. The experimental results highlighted that the inhibitory effect of ultrasound irradiation was lower following $15 \mathrm{~min}$ of exposure than after a shorter interval of $10 \mathrm{~min}$, emphasizing a complex, non-linear biological response. On the whole, the findings of this study indicated a complex mechanism for the inhibition of the radicular growth by ultrasound exposure. The ultrasound exposure of Triticum seeds may be used as a tool to either assess the effects of toxic agents following an increase in cell permeability, or to examine the synergistic action between ultrasounds and exposure to chemical mixtures that can promote physiological disruptions.
\end{abstract}

Correspondence to: Dr Dragoş Paul Mihai, Faculty of Pharmacy, 'Carol Davila' University of Medicine and Pharmacy, Traian Vuia 6, 020956 Bucharest, Romania

E-mail: dragosm1992@gmail.com

Key words: ultrasound, root length, phytobiological assay

\section{Introduction}

The inhibition of the root growth and the observation of the cytological parameters is a simple, and yet efficient method to evaluate new potential anticancer agents and can provide useful information about the genotoxicity risks (1). The Triticum root elongation test can be used to detect new compounds with anti-proliferative effects on cancer cells, particularly those compounds targeting tubulin and may be an effective tool with which to identify novel spindle inhibitors (2). The use of phytobiological tests has major advantages, such as a more rapid outcome, a simplified operative procedure and lower costs (3). Similar phytobiological tests are successfully used to evaluate the environmental effects in contaminated soils and wastewater caused by specific substances (4-6).

Ultrasounds are sound waves with frequencies higher than the upper audible limit of human hearing used for a wide range of applications. Over the years, it has been demonstrated that ultrasound exposure can have lethal effects on small organisms. The mechanism of cell destruction is mainly due to cavitated air bubbles and by heating, particularly at medium and high frequencies (7). Wells demonstrated the existence of a threshold level of ultrasound power below which the ultrasonic exposure of the planktonic crustacean Daphnia magna has no significant effect on their survival and of a second threshold level below which the mortality of Daphnia is only thermal and no mechanical damage can be observed (8). The acute lethality of ultrasound exposure on Daphnia has been demonstrated, with higher frequencies exerting a more potent effect than lower frequencies (9).

The use of ultrasound enhances the germination of certain seeds by the activation of various enzymes activity; however, to the best of our knowledge, no studies to date have been performed on the effects of root length. Ultrasound treatments have been reported to stimulate germination in different types of plants, such as Panicum virgatum (10), Zea mays (11), Sorghum bicolor (12) or Phaseolus vulgaris (13)

A major impediment of the bioassays involving plant cells is the low penetration of cell wall by the test substances, 
a process that could be facilitated by ultrasound irradiation. In the present study, the influence of the ultrasound exposure time to Triticum aestivum germination was evaluated, in order to determine whether this method may be used in conjunction with chemical compounds in the Triticum assay as a tool for the identification of novel anti-proliferative agents.

\section{Materials and methods}

The wheat caryopses (S.C.Adaflor S.R.L.) used were pre-soaked in distilled water for $24 \mathrm{~h}$ prior to the experiment. Ultrasonication was carried out using a working frequency of $37 \mathrm{kHz}$ (ElmaSonic S15H; Elma Schmidbauer GmbH). Groups containing 20 caryopses were exposed to a single continuous wave of ultrasounds for periods of $1,3,5,10,15$, 30 and $60 \mathrm{~min}$. The experiments were performed at room temperature. Following exposure, the caryopses were incubated for $24 \mathrm{~h}$ at $25^{\circ} \mathrm{C}$ in the absence of light in a climatic room chamber (Sanyo MLR351H; Sanyo). A negative control (untreated WITH ultrasound) was also prepared. The germination of the root was monitored and the embryonic radicle length was measured using application ImageJ version $1.46 \mathrm{r}$ (Wayne Rasband National Institutes of Health). The values of root length were expressed in $\mathrm{mm}$.

Each determination was performed in triplicate. The inhibitory effect (Efi\%) was calculated using the following formula:

$$
\text { Efi } \%=100-\frac{S}{C} \times 100
$$

where: $\mathrm{S}$ is the sample average of the root length $(\mathrm{mm})$, $\mathrm{C}$ is control sample average of the root length $(\mathrm{mm})$; and 100 indicates the results expressed as a percentage.

The distribution of the results (D'Agostino-Pearson normality test), statistically significant differences between replicates and samples (Kruskal-Wallis followed by Dunn's multiple comparison post hoc test) and the inhibitory effects vs. the logarithm of exposure time (least square method) were calculated using GraphPad Prism v.5.0 software (GraphPad Software). The statistical significance threshold was set to $\alpha=0.05$ for both distribution and comparison tests and a P-value $<0.05$ was considered to indicate a statistically significant difference. All analysis were performed against the negative control.

\section{Results}

The exposure of the wheat caryopses to the $37 \mathrm{kHz}$ constant ultrasounds irradiation produced an inhibitory effect depending on the exposure interval time. No statistical differences were registered between the lengths of the roots between replicates. The results of the D'Agostino-Pearson normality test, the mean values of each 20 seedlings root lengths, and the inhibitory effect are presented in Table I.

No statistically significant differences were registered between the samples exposed for 1 and $3 \mathrm{~min}$ and the negative control. Beginning with the 5-min exposure time, all results are statistically significant when compared with the negative control. An ultrasound exposure of up to 3 min can be used in the Triticum assay in conjunction with chemical compounds or plant extracts to improve their aqueous solubility, and therefore the cell permeability (14).

The distribution of each seedling root length value is represented in Fig. 1. The values obtained exhibited both a Gaussian and non-Gaussian distribution. Thus, the results were compared using the Kruskal-Wallis test, followed by Dunn's multiple comparison post hoc test.

A graphical representation of the inhibitory effects depending on the period of exposure is represented in Fig. 2. When a longer exposure time was used, physical or chemical damage occured caused by the pressure induced by the ultrasonic wave.

The resulted curve has an unexpected alternating, serrated shape. These phenomena may be explained by the large confidence intervals of the calculated mean or it may be caused by a complex biologic interaction. The most probable hypothesis is that the observed inhibitory effect is a sum of multiple opposing effects with different kinetics, the inhibitory effect being higher than the stimulatory one. The results are similar with those obtained by previous research on germination rates; ultrasound treatments $>5$ min had a negative impact on pepper germination rates. The same study indicated that ultrasound treatment increased the germination rates for chickpeas, wheat and watermelon seeds (15). A prolonged treatment time ( $\geq 7 \mathrm{~min}$ ) increased the percentage of destroyed embryos of seeds, which leads to decreased germination rates in Calanthe hybrids (16).

Short sonication $(3 \mathrm{~min})$ had no significant effect on germination compared to the control, although $\geq 5$ min sonication treatments led to a decline in germination. The inhibitory effect exceeded $50 \%$ following $10 \mathrm{~min}$ of exposure and was maximum at 30 and $60 \mathrm{~min}$, with no statistically significant differences between these time points. The exposure time of $30 \mathrm{~min}$ was sufficient for a total inhibitory effect. Similar to the $\mathrm{IC}_{50}$ value, a $50 \%$ inhibitory period of exposure was calculated by interpolation on inhibitory effect vs. logarithm of period of exposure curve (Fig. 3). The obtained value was 9.48 min with a $95 \%$ confidence interval ranging from 5.30 to $16.98 \mathrm{~min}$.

\section{Discussion}

Cytological assessments and root growth inhibition measurements are practical methods for assessing the biological activity of novel antineoplastic agents and acute toxicity in preliminary non-clinical studies $(2,17)$.

Exposure to ultrasound waves has been shown to have a negative impact on cell survival in small organisms (7). However, previous studies have highlighted that ultrasound irradiation enhances germination in several plant species $(10,18)$. Furthermore, ultrasonication can be used to increase cell wall permeability, as a means of removing one of the phytobiological assays limitations, facilitating the diffusion of the investigating compounds.

This study aimed to assess the outcomes of different time intervals of exposure with regard to the possible applications of ultrasound irradiation in the Triticum bioassay. Wheat caryopses were exposed to a $37 \mathrm{kHz}$ constant ultrasound waves 
Table I. Results of the Triticum test.

\begin{tabular}{|c|c|c|c|c|c|c|}
\hline \multirow[b]{2}{*}{ Time of determination (min) } & \multirow[b]{2}{*}{ Replicate } & \multicolumn{2}{|c|}{ D'Agostino-Pearson normality test } & \multirow[b]{2}{*}{ Mean $(\mathrm{mm})$} & \multirow[b]{2}{*}{ Standard deviation } & \multirow[b]{2}{*}{ Efi\% } \\
\hline & & P-value & Passed normality test & & & \\
\hline \multirow[t]{3}{*}{1} & $\mathrm{~S} 1.1$ & 0.1344 & Yes & 9.08 & 3.20 & 11.47 \\
\hline & $\mathrm{S} 1.2$ & 0.0825 & Yes & 10.01 & 3.69 & \\
\hline & $\mathrm{S} 1.3$ & 0.4196 & Yes & 9.02 & 3.53 & \\
\hline \multirow[t]{3}{*}{3} & S3.1 & 0.0017 & No & 10.22 & 3.64 & 10.31 \\
\hline & S3.2 & 0.0086 & No & 9.37 & 2.63 & \\
\hline & S3.3 & 0.1524 & Yes & 8.89 & 3.36 & \\
\hline \multirow[t]{3}{*}{5} & S5.1 & 0.0012 & No & 5.56 & 3.91 & 43.76 \\
\hline & S5.2 & 0.0582 & Yes & 7.63 & 3.46 & \\
\hline & S5.3 & 0.1327 & Yes & 4.66 & 3.87 & \\
\hline \multirow[t]{3}{*}{10} & $\mathrm{~S} 10.1$ & 0.0221 & No & 4.76 & 3.82 & 58.88 \\
\hline & $\mathrm{S} 10.2$ & 0.0941 & Yes & 3.74 & 3.79 & \\
\hline & $\mathrm{S} 10.3$ & 0.0408 & No & 4.56 & 3.47 & \\
\hline \multirow[t]{3}{*}{15} & S15.1 & 0.122 & Yes & 6.18 & 3.17 & 43.69 \\
\hline & S15.2 & 0.1734 & Yes & 5.87 & 3.40 & \\
\hline & S15.3 & 0.3571 & Yes & 5.83 & 3.62 & \\
\hline \multirow[t]{3}{*}{30} & $\mathrm{~S} 30.1$ & 0.0545 & Yes & 0.40 & 0.66 & 89.53 \\
\hline & $\mathrm{S} 30.2$ & $<0.0001$ & No & 1.21 & 2.50 & \\
\hline & $\mathrm{S} 30.3$ & 0.0009 & No & 1.71 & 2.90 & \\
\hline \multirow[t]{3}{*}{60} & S60.1 & $<0.0001$ & No & 1.50 & 2.82 & 88.88 \\
\hline & S60.2 & $<0.0001$ & No & 1.26 & 2.35 & \\
\hline & S60.3 & $<0.0001$ & No & 0.77 & 1.81 & \\
\hline \multirow[t]{3}{*}{ Control } & $\mathrm{C} 1$ & $<0.0001$ & No & 10.47 & 3.21 & - \\
\hline & $\mathrm{C} 2$ & 0.0002 & No & 10.08 & 3.17 & \\
\hline & $\mathrm{C} 3$ & 0.5268 & Yes & 11.20 & 2.29 & \\
\hline
\end{tabular}

and the embryonic radicle length was measured following $24 \mathrm{~h}$ of incubation. This frequency was selected as the majority of laboratory ultrasound baths operate in the $35-40 \mathrm{kHz}$ range. A time-dependent inhibition of root development was observed, with the mean length of the radicle being statistically significantly different from the negative control only after 5 min of ultrasound exposure.

The serrated shaped of root length - inhibitory effect over time curve indicated that the inhibitory effect of ultrasound irradiation was lower following 15 min of exposure than after a shorter interval of $10 \mathrm{~min}$, emphasizing a complex, non-linear biological response, probably caused by a perturbation of a complex balance between the kinetics of inhibitory and stimulatory mechanisms. Moreover, ultrasound irradiation of wheat caryopses registered a plateau effect after $30 \mathrm{~min}$, the inhibitory effect (Efi\%) being maximal and approximately constant at both the 30 and 60 min time intervals. Similar studies have demonstrated that ultrasound exposure of at least 5 min decreases pepper germination rates, while ultrasonication $>7$ min negatively affects Calanthe hybrids embryos $(15,16)$. The interpolation of the inhibitory effect-logarithm of period of exposure curve yielded a $50 \%$ inhibitory period of ultrasonic exposure of $9.49 \mathrm{~min}$.

Considering there was no significant impact recorder on germination rates after 1 and 3 min of ultrasonic irradiation, it can be considered that an ultrasound exposure of $\leq 3$ min of Triticum aestivum seeds opportune for increasing solubility and cell permeation of novel antineoplastic candidates for preliminary phytobiological assays of anti-proliferative properties.

High-intensity focused ultrasounds are used in humans in a non-invasive manner for therapeutic purposes, surgery and enhanced delivery of chemotherapy and biologic drugs and can generate side effects at different levels (lungs, intestines, urinary tract, heart etc.) (19). Moreover, a new paradigm has emerged stating that real-life risk simulations of toxicological effects in humans should take into account long-term exposure with mixtures of synergistic chemicals at low doses (20-22). Several studies already showed the health risks ofter combined exposure to mixtures of xenobiotics after long-term, even if the doses for individual chemicals in the mixture are considered safe (23-27). High-intensity focused ultrasounds are used in association with other chemicals and this association in real-life cases can lead to unpredictive toxic effects that should be investigated. Thus, the ultrasound exposure of Triticum seeds may be used as a tool to either assess the effects of toxic agents after cell permeability increase, or to examine the synergistic action between ultrasounds and exposure to chemical mixtures that can promote physiological disruptions. 

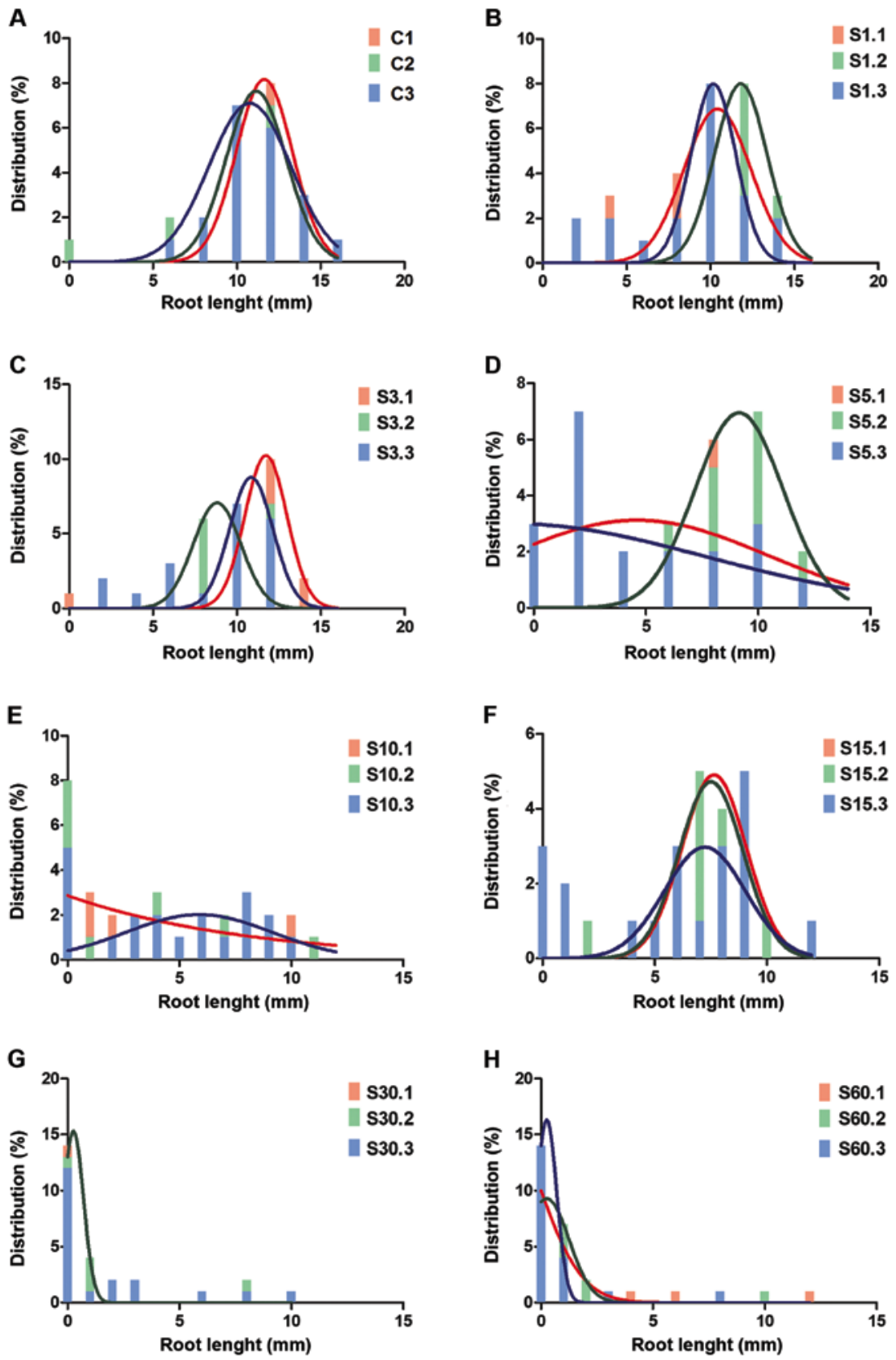

Figure 1. Distribution - root length histograms of (A) negative control and samples exposed to ultrasounds for (B) $1 \mathrm{~min}$, (C) $3 \mathrm{~min}$, (D) $5 \mathrm{~min}$, (E) $10 \mathrm{~min}$, (F) $15 \mathrm{~min}$, (G) $30 \mathrm{~min}$ and (H) $60 \mathrm{~min}$.

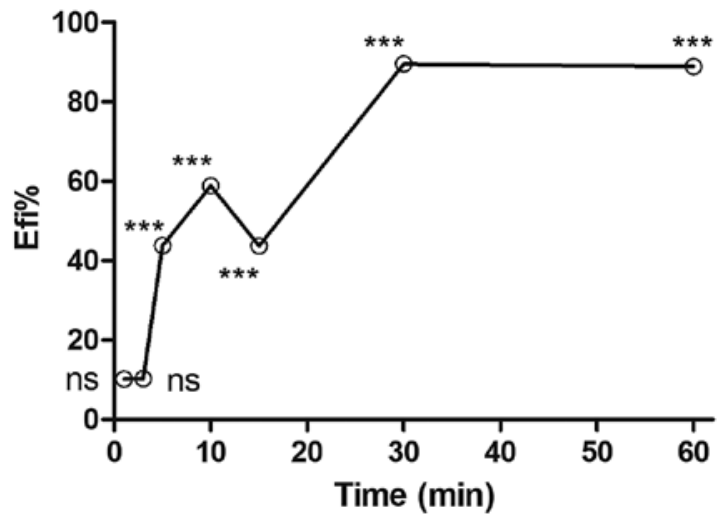

Figure 2. Influence of time of ultrasound irradiation on radicle development inhibition. Efi\%, inhibitory effect as a percentage. Statistical significance is indicates as follows: $n s$, not significant $(\mathrm{P}>0.05) ;{ }^{* * *} \mathrm{P}<0.001$ significant.

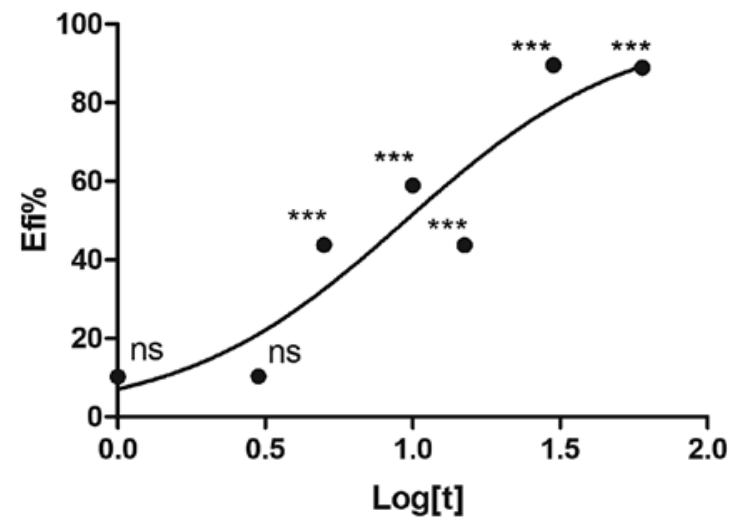

Figure 3. Inhibitory effect of ultrasound exposure dependent on time. Efi\%, inhibitory effect as a percentage. Statistical significance is indicates as follows: ns, not significant $(\mathrm{P}>0.05) ;{ }^{* * *} \mathrm{P}<0.001$ significant. 
In conclusion, the present study evaluated the influence of ultrasounds on the length of the root using Triticum bioassay. The results indicated a significant inhibitory effect of ultrasounds following $5 \mathrm{~min}$ and no differences in the ultrasonic treatment performed for $\leq 3 \mathrm{~min}$. The increase in solubility for the plant extracts using short ultrasound irradiation may prove to be a tool in future research for the identification of potential anticancer agents and for the assessment of the synergistic action between longer exposure times and chemical mixtures.

\section{Acknowledgements}

Not applicable.

\section{Funding}

No funding was received.

\section{Availability of data and materials}

All data generated and analyzed are included in the published article.

\section{Authors' contributions}

OTO, GMN and AT were involved in the conception and design of the study. IMP, OMP, CES, GN, DPM, AT, DAS and OTO were involved in the investigative aspects of the study. OTO and GMN were involved in the study methodology. GN, DPM, OTO and GMN were involved in the writing of the original draft. DPM, OTO, AT, DAS and GMN reviewed and edited the manuscript. All authors have read and approved the final manuscript.

\section{Ethics approval and consent to participate}

Not applicable.

\section{Patient consent for publication}

Not applicable.

\section{Competing interests}

DAS is the Managing Editor of the journal, but had no personal involvement in the reviewing process, or any influence in terms of adjudicating on the final decision, for this article. The other authors declare that they have no competing interests.

\section{References}

1. Nitulescu GM, Draghici C and Olaru OT: New potential antitumor pyrazole derivatives: Synthesis and cytotoxic evaluation. Int J Mol Sci 14: 21805-21818, 2013.

2. Olaru OT, Zanfirescu A, Nitulescu GM, Nitulescu G, Dinu-Pirvu CE, Anuta V, Tsatsakis A, Spandidos DA, Margina D and Seremet OC: Predictive power of the Triticum root elongation test for the assessment of novel anti proliferative therapies. Int J Mol Med 44: 16-24, 2019.
3. Margină D, Olaru OT, Ilie M, Grădinaru D, GuȚu C, Voicu S, Dinischiotu A, Spandidos DA and Tsatsakis AM: Assessment of the potential health benefits of certain total extracts from Vitis vinifera, Aesculus hyppocastanum and Curcuma longa. Exp Ther Med 10: 1681-1688, 2015.

4. Guzman-Rangel G, Montalvo D and Smolders E: Pronounced Antagonism of Zinc and Arsenate on Toxicity to Barley Root Elongation in Soil. Environments 5: 83, 2018.

5. Zhu G, Jiang B, Yang G, Li J and Ma Y: Toxicity thresholds based on EDTA extractable nickel and barley root elongation in Chinese soils. Int J Environ Res Public Health 15: 15, 2018.

6. Jaskowiak J,Kwasniewska J,Milewska-Hendel A,KurczynskaEU, Szurman-Zubrzycka M and Szarejko I: Aluminum Alters the Histology and Pectin Cell Wall Composition of Barley Roots. Int J Mol Sci 20: 20, 2019.

7. Suslick KS, Hammerton DA and Cline RE: Sonochemical hot spot. J Am Chem Soc 108: 5641-5642, 1986.

8. Wells PN: The effect of ultrasonic irradiation on the survival of Daphnia magna. J Exp Biol 49: 61-70, 1968.

9. Lürling $\mathrm{M}$ and Tolman Y: Effects of Commercially Available Ultrasound on the Zooplankton Grazer Daphnia and Consequent Water Greening in Laboratory Experiments. Water 6: 3247-3263, 2014.

10. Wang Q, Chen G, Yersaiyiti H, Liu Y, Cui J, Wu C, Zhang Y and He X: Modeling analysis on germination and seedling growth using ultrasound seed pretreatment in switchgrass. PLoS One 7: e47204, 2012.

11. Karabascheff N, Metev V and Kutov T: On the effect of ultrasonic waves on the germination of corn. C R Acad Bulg Sci 19: 305-312, 1966 (In German).

12. Patero T and Augusto PED: Ultrasound (US) enhances the hydration of sorghum (Sorghum bicolor) grains. Ultrason Sonochem 23: 11-15, 2015.

13. Ghafoor M, Misra NN, Mahadevan K and Tiwari BK: Ultrasound assisted hydration of navy beans (Phaseolus vulgaris). Ultrason Sonochem 21: 409-414, 2014.

14. Gîrd CE, Costea T, Duţu LE, Popescu ML and Nencu I: Phytochemical and phytobiological research regarding roots of Heracleum Sphondylium L. and H. Palmatum Baumg. Trends Toxicol Relat Sci 1: 84-93, 2017.

15. Goussous SJ, Samarah NH, Alqudah AM and Othman MO: Enhancing seed germination of four crop species using an ultrasonic technique. Exp Agric 46: 231-242, 2010.

16. Shin YK, Baque MA, Lee EJ and Paek KY: Effects of activated charcoal, plant growth regulators and ultrasonic pre-treatments on in vitro germination and protocorm formation of Calanthe hybrids. Aust J Crop Sci 5: 582-588, 2011.

17. Wu M, Wang X, Jia Z, De Schamphelaere K, Ji D, Li X and Chen X: Modeling acute toxicity of metal mixtures to wheat (Triticum aestivum L.) using the biotic ligand model-based toxic units method. Sci Rep 7: 9443, 2017.

18. Sadeghianfar P, Nazari M and Backes G: Exposure to Ultraviolet (UV-C) Radiation Increases Germination Rate of Maize (Zea maize L.) and Sugar Beet (Beta vulgaris) Seeds. Plants 8: 49, 2019.

19. Izadifar Z, Babyn P and Chapman D: Mechanical and Biological Effects of Ultrasound: A Review of Present Knowledge. Ultrasound Med Biol 43: 1085-1104, 2017.

20. Tsatsakis AM, Docea AO and Tsitsimpikou C: New challenges in risk assessment of chemicals when simulating real exposure scenarios; simultaneous multi-chemicals' low dose exposure. Food Chem Toxicol 96: 174-176, 2016.

21. Margina D, Nitulescu GM, Ungurianu A, Mesnage R, Goumenou M, Sarigiannis DA, Aschner M, Spandidos DA, Renieri EA, Hernández AF, et al: Overview of the effects of chemical mixtures with endocrine disrupting activity in the context of real life risk simulation (RLRS): An integrative approach (Review). World Acad Sci J 1: 157-164, 2019.

22. Tsatsakis AM, Kouretas D, Tzatzarakis MN, Stivaktakis P, Tsarouhas K, Golokhvast KS, Rakitskii VN, Tutelyan VA, Hernandez AF, Rezaee R, et al: Simulating real-life exposures to uncover possible risks to human health: A proposed consensus for a novel methodological approach. Hum Exp Toxicol 36: 554-564, 2017.

23. Fountoucidou P, Veskoukis AS, Kerasioti E, Docea AO, Taitzoglou IA, Liesivuori J, Tsatsakis A and Kouretas D: A mixture of routinely encountered xenobiotics induces both redox adaptations and perturbations in blood and tissues of rats after a long-term low-dose exposure regimen: The time and dose issue. Toxicol Lett 317: 24-44, 2019. 
24. Tsatsakis A, Docea AO, Constantin C, Calina D, Zlatian O, Nikolouzakis TK, Stivaktakis PD, Kalogeraki A, Liesivuori J, Tzanakakis G, et al: Genotoxic, cytotoxic, and cytopathological effects in rats exposed for 18 months to a mixture of 13 chemicals in doses below NOAEL levels. Toxicol Lett 316: 154-170, 2019.

25. Tsatsakis A, Tyshko NV, Docea AO, Shestakova SI, Sidorova YS, Petrov NA, Zlatian O, Mach M, Hartung T and Tutelyan VA: The effect of chronic vitamin deficiency and long term very low dose exposure to 6 pesticides mixture on neurological outcomes - A real-life risk simulation approach. Toxicol Lett 315: 96-106, 2019

26. Tsatsakis AM, Docea AO, Calina D, Buga AM, Zlatian O, Gutnikov S, Kostoff RN and Aschner M: Hormetic Neurobehavioral effects of low dose toxic chemical mixtures in real-life risk simulation (RLRS) in rats. Food Chem Toxicol 125: 141-149, 2019.
27. Docea AO, Gofita E, Goumenou M, Calina D, Rogoveanu O, Varut M, Olaru C, Kerasioti E, Fountoucidou P, Taitzoglou I, et al: Six months exposure to a real life mixture of 13 chemicals' below individual NOAELs induced non monotonic sex-dependent biochemical and redox status changes in rats. Food Chem Toxicol 115: 470-481, 2018.

(i)(2) This work is licensed under a Creative Commons

EY NC NO Attribution-NonCommercial-NoDerivatives 4.0 International (CC BY-NC-ND 4.0) License. 